\title{
EDUFAM. Mejora del sistema educativo a través de la formación de familiares de grupos vulnerables. Plan Nacional $I+D+i$ (2014-2016).
}

Entitat que finança: Plan Nacional I+D+I. Ministerio de Economía i Competitividad.

Durada: 2014-16

\section{Resumen de la investigación}

Las estadísticas Internacionales muestran que la distancia en cuanto a rendimiento educativo entre los niños y niñas de familias académicas y los de familias noacadémicas es una realidad que se da en todo el mundo. Uno de los objetivos de Europa 2020 consiste en hacer realidad el aprendizaje a lo largo de toda la vida (Comisión Europea, 2010). Sin embargo, las evidencias obtenidas hasta el momento reflejan que las personas y colectivos que menos participan en formación permanente y aprendizaje a lo largo de la vida son precisamente aquellos que tienen menos acreditaciones académicas y se encuentran en situaciones de vulnerabilidad, siendo por lo tanto aquellas para quienes resultaría más importante y necesario. Se ha identificado que la formación de familiares ha sido una de las actuaciones educativas de éxito para la mejora de resultados escolares de todo el alumnado y de convivencia (INCLUD-ED Consortium, 2009).

El proyecto EDU-FAM pretende contribuir al diálogo entre la ciencia y las necesidades sociales para dar una respuesta realista y eficaz a los retos planteados tanto por el Plan Estatal de Investigación Científica y Técnica y de Innovación 2013 - 2016, como Horizon 2020 vinculado a la mejora de un sistema educativo de calidad. Ofreciendo evidencias científicas de cómo y de qué manera la formación de familiares en grupos vulnerables contribuye al éxito escolar de todo el alumnado y a la participación de todas las personas en educación a lo largo de la vida, e implicando activamente a todos los actores afectados para facilitar su transferencia a diferentes niveles del sistema educativo. 
La comunidad científica internacional ya ha identificado la formación de familiares como una actuación educativa que mejora el éxito escolar del alumnado y la cohesión social. Por ello, el objetivo general del proyecto EDU-FAM consiste en aportar conocimiento científico multidisciplinar sobre las características de la implementación de formación de familiares en grupos vulnerables en diferentes contextos (incluyendo experiencias internacionales) y profundizar en los beneficios para el alumnado y las propias personas adultas, creando sinergias entre actores y facilitando la transferencia de resultados para la mejora del sistema educativo.

Con una duración de tres años, se seleccionarán 8 actuaciones de formación de familiares españolas que hayan aportado evidencias de su impacto, en las que se pretende realizar: un estudio de caso en cada una mediante técnicas cualitativas y cuantitativas para identificar características comunes y formas de superar las resistencias y dificultades encontradas en su implementación; trabajo de campo cualitativo con familias, alumnado y profesionales para analizar los beneficios en el alumnado y en las propias personas familiares; y un análisis longitudinal de un/a familiar participante en cada una de las actuaciones, para profundizar en el estudio del impacto de la formación de familiares a lo largo del tiempo. Todos los centros atenderán a población y alumnado de grupos vulnerables, con especial mención a familias gitanas (al menos en 2 centros). Los resultados obtenidos se complementarán con dos estudios de caso de experiencias exitosas internacionales y se contrastarán con otras seis experiencias internacionales que se están desarrollando en Europa, Estados Unidos y América Latina, a través de un grupo de discusión en cada caso.

\section{Equipo de investigación:}

Investigador principal: Aitor Gómez. Universitat Rovira i Virgili

Miembros del equipo:

Adriana Aubert Simón: Universitat de Barcelona

Joan Cabré Olivé: Universitat Rovira i Virgili

María Pilar Colás Bravo: Universidad de Sevilla

Miquel Àngel Comas Parras: Universidad Internacional de la Rioja.

Javier Díez Palomar: Universitat de Barcelona

Alfonso García Monge: Universidad de Valladolid

Carme Garcia Yeste: Universitat Rovira i Virgili

Montse Guasch Garcia: Universitat Rovira i Virgili

Fernando Macías Aranda: Universitat de Barcelona

Noemí Martín Casabona: Universitat Rovira i Virgili 
Maria Padrós Cuxart: Universitat de Barcelona

Sandra Racionero Plaza: Universidad de Loyola Andalucia

Laura Ruiz Eugenio: Universitat de Barcelona

Ma Ángeles Serrano: Universitat de Barcelona

Joana M. Tierno Garcia: Universitat Rovira i Virgili

Luís Torrego Egido: Universidad de Valladolid

\section{Equipo internacional:}

Kyung Hi Kim: Kyungnam University (Corea del Sur)

Donatila Ferrada: Universidad Católica de la Santísima Concepción (Chile)

Maria Vieites. Instituto Natura (Brasil) 